Document downloaded from:

http://hdl.handle.net/10251/44276

This paper must be cited as:

Villegas, M.; Paredes Palacios, R. (2007). Face Recognition in Color Using Complex and Hypercomplex Representation. En Pattern Recognition and Image Analysis: Third Iberian Conference, IbPRIA 2007, Girona, Spain, June 6-8, 2007, Proceedings, Part I. Springer Verlag (Germany). 217-224. doi:10.1007/978-3-540-72847-4_29.

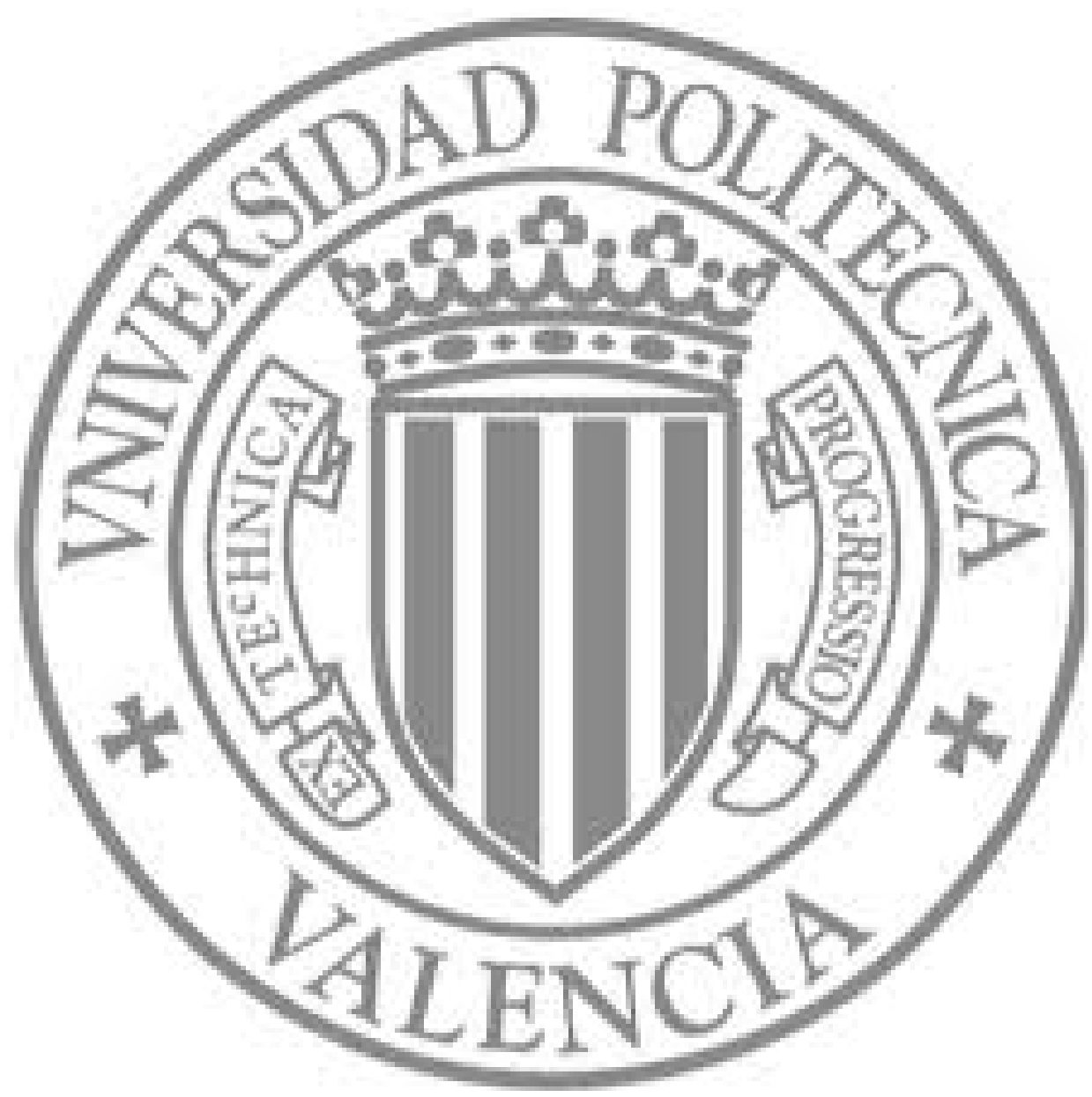

The final publication is available at

http://link.springer.com/chapter/10.1007/978-3-540-72847-4_29

Copyright Springer Verlag (Germany) 


\title{
Face Recognition in Color Using Complex and Hypercomplex Representations *
}

\author{
Mauricio Villegas and Roberto Paredes \\ Instituto Tecnológico de Informática \\ Universidad Politécnica de Valencia \\ Camino de Vera s/n, Edif. 8G Acc. B 46022 Valencia (Spain) \\ \{mvillegas, rparedes\}@iti.upv.es
}

\begin{abstract}
Color has plenty of discriminative information that can be used to improve the performance of face recognition algorithms, although it is difficult to use it because of its high variability. In this paper we investigate the use of the quaternion representation of a color image for face recognition. We also propose a new representation for color images based on complex numbers. These two color representation methods are compared with the traditional grayscale and RGB representations using an eigenfaces based algorithm for identity verification. The experimental results show that the proposed method gives a very significant improvement when compared to using only the illuminance information.
\end{abstract}

\section{Introduction}

Humans have a high performance for recognizing faces even when they are in grayscale. However resent research on face recognition by humans indicates that color information is very important in certain circumstances, for example when images have an extremely low resolution [1].

The use of color introduces new sources of variability that makes the problem harder and more expensive to resolve. But despite these problems, if humans recognize faces better when they are in color, then there is no doubt that the color contains very useful information that can help improve the accuracies of current systems $[1-3]$.

The main topic that we want to address in this paper is how to represent the color images in order to take advantage of all the information it holds. Moreover we are going to apply light normalization techniques to the proposed color representations in order to avoid the effect of illumination and color variability.

This paper is organized as follows: Section 2 discusses color image representations in general, describes the two representation methods being compared and two baseline techniques. Section 3 describes the normalization methods used in the experiments. Section 4 presents the algorithm used in the experiments and

\footnotetext{
* Work supported by the the Spanish Project DPI2004-08279-C02-02 and the Generalitat Valenciana - Consellería d'Empresa, Universitat i Ciència under an FPI scholarship.
} 
the adaptations to the new color representations. Section 5 shows the results of the experiments carried out. Finally section 6 draws the conclusions and directions for future research.

\section{Representing Color Images}

There are many ways in which a color image can be represented mathematically. The straightforward representation is to link the three color components so the dimensionality of the feature vector is three times the dimensionality of the grayscale version. We will use this representation and the grayscale representation as baselines in our experiments.

In this paper we compare two methods for representing color images. The first one using quaternion numbers, and a proposed one using complex numbers. The benefit of these representations is that they use the color information while the pixel structure is preserved.

\subsection{Quaternion Representation of Images}

The quaternions can be seen as a generalization of complex numbers to four dimensions [4]. Any quaternion can be written using four real numbers as

$$
q=q_{r}+q_{i} \mathbf{i}+q_{j} \mathbf{j}+q_{k} \mathrm{k}
$$

where $q_{r}, q_{i}, q_{j}, q_{k} \in \mathbb{R}$ and $\mathrm{i}, \mathrm{j}, \mathrm{k}$ are three different imaginary units related by

$$
i^{2}=j^{2}=k^{2}=i j k=-1
$$

The set of quaternion numbers is commonly denoted by $\mathbb{H}$ named after the person that proposed them W. R. Hamilton [4]. For the quaternions most of the algebraic properties are the same as for real or complex numbers. A particular property that distinguishes them from real or complex numbers is that multiplication is not commutative. For a more detailed review of the quaternion properties refer to [5].

The quaternions have been proposed for modelling color images [6] which has been successfully applied in digital image processing [7-9], although it has not been used for image recognition. This approach represents each pixel of an image using a single quaternion, the real part is set to zero and the three color components are assigned to the imaginary parts. The resulting representation is a quaternion vector of dimension the same as the number of pixels in the image.

The fact that the algebraic properties of the quaternion are similar to the real numbers and has a mathematical background, makes them very attractive to be used for representing color. In fact, most of the algorithms that exist for grayscale images can be easily generalized to the quaternion domain. 


\subsection{Complex Representation of Images}

There is major drawback when representing color images as quaternions and using the generalized algorithms from the grayscale version. The problem is that the algorithms tend to be more computationally expensive. For example a single multiplication of two scalars in the quaternion domain in general requires sixteen distinct multiplications. It is understandable that the algorithms used for color images could be more expensive than the ones used for grayscale images. Although it would be desirable to find algorithms that can be used for color images without increasing considerably their cost.

In this section we propose to represent color images as a complex valued vector, where each pixel is represented by a single complex number. This representation also makes possible to generalize the grayscale algorithms to color, while having a lower cost than the quaternion representation. Because a complex number is composed of only two values, we need to find a way to represent the color components using two numbers. To do this we are going to define a new color space, the PCA color space.

It is complicated to define a two component color space that can be used for image recognition in general. But if we consider a specific problem, for example in this case the images are only from human faces, a better color space can be defined. It is known that the possible colors of faces lie on a well defined region of the color space [10]. If we use a dimensionality reduction technique such as Principal Component Analysis (PCA), we are capable of reducing the three color components to two while making sure that we retain most of the original color information. The first and second principal components are assigned to the real and imaginary parts respectively, and the third principal component is ignored.

This PCA color space depends on the task being considered and it needs to be calculated using the training data.

\section{Illumination Normalization}

In a face recognition system one of the most difficult properties to achieve is illumination invariance. This means the performance of the system must not be affected by the amount of lighting conditions. One possibility to reduce this variability is to process the image prior to the recognition stage aiming to obtain a representation invariant to the illumination conditions. In the face recognition context this is commonly called illumination normalization, for color images this is also called color constancy.

Several illumination normalization techniques exist [11-13]. A very simple one that gives good results is to locally make the mean of the image to zero and the variance to one [14]. Here we propose to extend this algorithm to color images by normalizing each component of the color space independently. Normalizing an image $I$ globally for each color component $c$ would be

$$
\hat{I}_{c}=\frac{I_{c}-\mu_{c}}{\sigma_{c}}
$$


where $\mu_{c}$ and $\sigma_{c}$ is the mean and the standard deviation of the color component $c$ respectively.

The local normalization is analogous to the global one, the difference is that the normalization function is applied locally. By applying a function locally we mean the following. A region of the image of size $w \times w$, is selected and the normalization function is applied to it. This process is repeated by moving the selection window pixel by pixel all over the image. Because the windows overlap, the final pixel value is the average of all the results for that particular pixel.

\section{Face Verification Algorithm}

In a face verification system a person claims a certain identity and a decision is taken whether it is or it is not the true identity. Features are extracted from the person's face image and are compared with the the model of the claimed identity. The client is accepted or rejected depending on a threshold.

The objective of the chosen algorithm is to assess if the representations described previously are adequate to be used in face verification and if they give a considerable improvement when compared to using the baseline representations. To do this we are going to define a simple algorithm that works analogously in the real domain for grayscale and linked color components images, and in the complex and quaternion domains for color images.

\subsection{Eigenfaces}

When images are represented by a single vector, as in our case, the original dimensionality of the feature vectors is very high. For this reason most of the recognition algorithms use some sort of dimensionality reduction technique before the classifier. A very popular technique is Principal Component Analysis (PCA), which in the context of face recognition it is known as eigenfaces [15]. Eigenfaces has become a general baseline for face recognition, therefore it is the one that we used. For the classifier we used the nearest neighbor using the Euclidean distance.

Because we are representing the images using complex and quaternion vectors, we also need PCA in these domains. PCA has already been generalized to quaternion vectors [8]. For complex vectors the generalization can be seen as a special case of quaternion PCA.

At the moment there is no mathematical software that has full support for quaternions. So in order to do a singular value decomposition of a quaternion matrix, which is needed for doing PCA, we have to use an isomorphism. This isomorphism is explained in [16].

\section{Experiments}

The corpora used in the experiments are the frontal datasets of the XM2VTS database [17], referred to as CDS001 and CDS006. Each dataset corresponds 
to one of the two shots taken at each of the sessions. Four session where taken distributed over a period of four months. In total there are eight images for each of the 295 subjects. The faces where manually cropped and resized to $24 \times 24$ pixels using the eye coordinates that are publicly available.

Verification on the XM2VTS database is evaluated using the Lausanne protocol [18]. This protocol specifies two experimentation configurations that state which images are to be used as training, evaluation and test. The protocol also specifies the measurements to be used so that the performance of different algorithms can be compared. Apart from the training, evaluation and test sets specified on the protocol configuration I, we generated nine more randomized sets. With these sets, the experiments where repeated ten times in order to make the results more reliable.

\subsection{The Eigenfaces}

Figure 1 shows the mean face and the first seven eigenfaces for each representation method without illumination normalization. The most interesting thing to note is that for all the methods the eigenfaces are quite similar. The eigenfaces can be seen as directions of maximum variability of the data. If these directions do not change by introducing color information, this indicates that there is a correlation between the global structure of the face and the color content. This result can be observed by considering that the race of a person not only indicates a particular skin color, but also a particular facial structure.

\section{$5.2 \quad$ Verification Results}

Figure 2 shows some graphs of the total error rates (see [18] for details) for the evaluation set with respect to the degrees of freedom. By degrees of freedom it is meant the amount of real numbers used to represent the feature vector. $D^{\prime}$ principal components produces $D^{\prime}, D^{\prime} / 2$ or $D^{\prime} / 4$ numbers for real, complex and quaternion vectors respectively. We have plotted the graphs this way because for the same degrees of freedom all of the methods have approximately the same verification computational cost. Three different graphs are obtained according to the three different preprocessing techniques: none, global and local normalization.

When there is no normalization the color representations give lower error rates although analyzing the confidence intervals it can be seen that they are not statistically better than gray. Using global normalization the color methods are considerably better than gray. The two methods rgb and cplx reach a minimum at 64 degrees of freedom, for quat the behavior is somewhat different, the minimum is located at 256 degrees of freedom.

For local normalization, the curves show the results taking the best normalization window size $w$ for each method. Although we expected a general improvement in all of the methods [14], for gray the errors only decrease slightly and for rgb and quat the errors even increase. In contrast the errors for cplx improve considerably. 

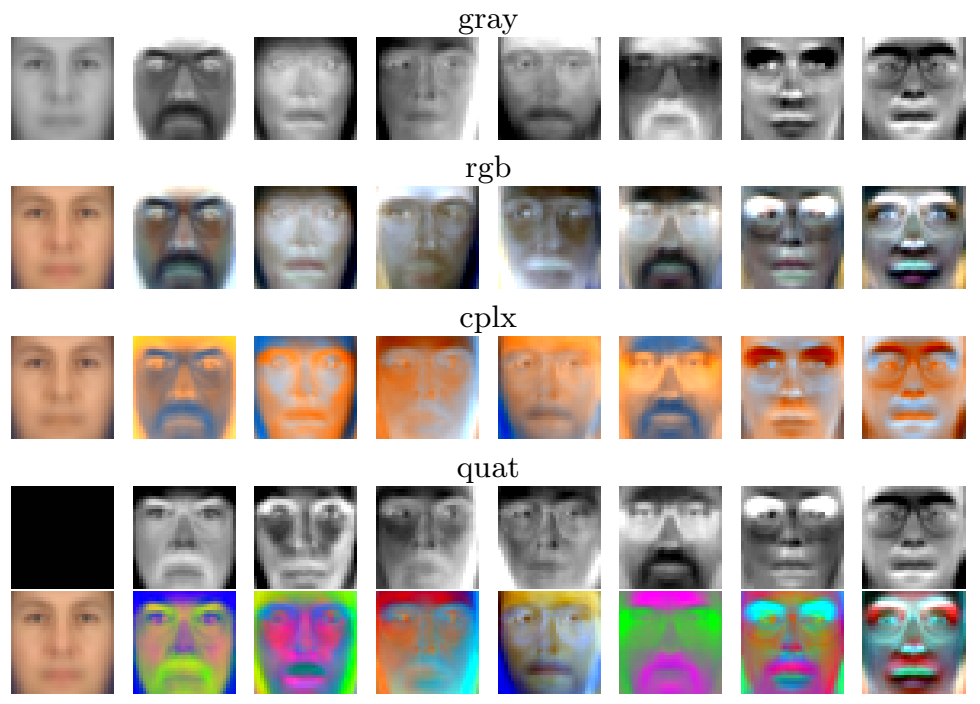

Fig. 1. The mean face (left column) and the first eigenfaces (columns 2 to 8) ordered in decreasing eigenvalue for each representation method. For quat the first row is the real part and the second row are the imaginary parts represented as a color image.

The final graph shows the best result for each of the methods. The optimum parameters for each method were: for gray $d=64$ and local normalization $w=22$, for $\operatorname{rgb} d=64$ and global normalization, for cplx $d=64$ and local normalization $w=14$, and for quat $d=256$ and global normalization. Only cplx gives a significant improvement with respect to gray and rgb.

Table 1. Error rates for the evaluation and test sets for each representation method, the $95 \%$ confidence intervals and the relative improvement with respect to gray.

\begin{tabular}{||c||c|c|c||c|c|c||}
\hline \multicolumn{1}{||c||}{ Method } & \multicolumn{3}{c||}{ Evaluation Set } & \multicolumn{3}{c||}{ Test Set } \\
\cline { 2 - 7 } & TER & $95 \%$ conf. int. & Improvement & TER & $95 \%$ conf. int. & Improvement \\
\hline gray & 19.0 & $18.4-19.7$ & & 17.3 & $16.4-18.1$ & \\
rgb & 17.0 & $16.4-17.7$ & $10.5 \%$ & 16.3 & $15.4-17.3$ & $5.3 \%$ \\
cplx & $\mathbf{1 4 . 1}$ & $13.4-14.2$ & $\mathbf{2 6 . 2 \%}$ & $\mathbf{1 2 . 9}$ & $12.1-13.7$ & $\mathbf{2 5 . 2 \%}$ \\
quat & 17.0 & $16.4-17.7$ & $10.5 \%$ & 16.5 & $15.7-17.3$ & $4.4 \%$ \\
\hline
\end{tabular}

Table 1 shows the error rates for each method with the optimum parameters, for both the evaluation and test sets. The relative improvement of the color representations with respect to gray is also presented.

The relative improvement obtained using the complex representation is quite significant. This suggest that the color can be used effectively as a discriminative feature in face recognition. The same improvement is seen in both the evaluation 
No normalization

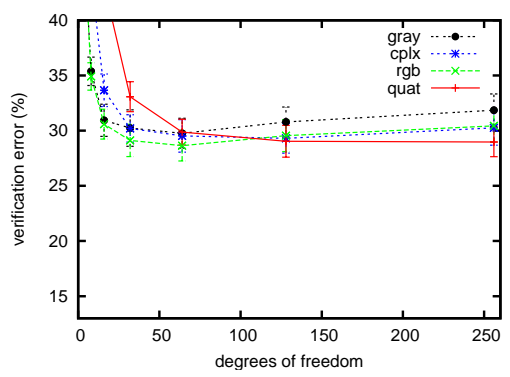

Local normalization

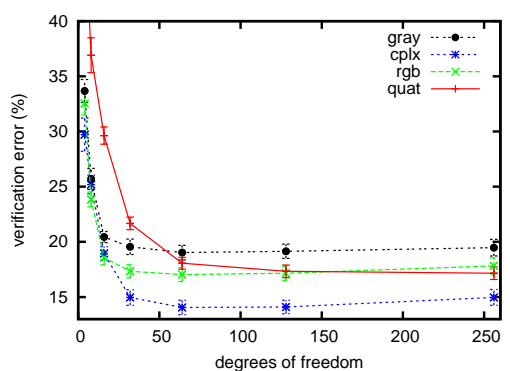

Global normalization

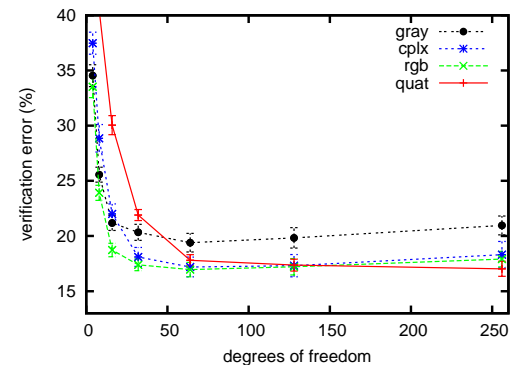

Best parameters

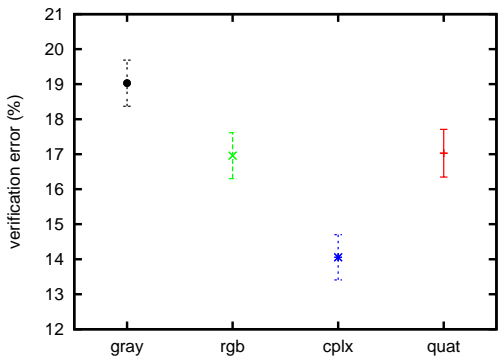

Fig. 2. The first three graphs are for the total error rates for the evaluation set [18] with respect to the degrees of freedom for the different preprocessing schemes. The last graph are the error rates for the preprocessing parameters that give the best result in each method. Error bars indicate the $95 \%$ confidence intervals.

and the test set so the representation also had the characteristic of generalizing well to new data. The results obtained using the quaternion representation are disappointing, comparing with rgb, there is practically no improvement for the evaluation set and for the test it is actually worse.

\section{Conclusions and future work}

In this paper we studied two possible ways of representing color images for the purpose of being used in face recognition. These where tested using the XM2VTS database for face verification.

The first one is based on the quaternion model of a color image, which has been successfully used in digital image processing. It was seen that although it could improve the system performance, it requires parameters that make the algorithm very expensive.

The results from the second representation, using complex numbers, show a significant improvement. This suggests that color is an important discriminative feature that can be used to improve considerably the accuracy of a system when compared to using only the illuminance information. Although this representa- 
tion gives a big improvement, it is not conclusive on whether the gain is due to the actual representation or to the color space and illumination normalization methods used.

As future research, we are going to use the same ideas presented here to extend other grayscale algorithms to color, so that we can achieve competitive recognition accuracies. Also, we should use other face databases that have a less constrained environment to see if the improvement keeps being significant.

Other research topics to explore is to find new color spaces and normalization techniques, that like the proposed PCA color space and local normalization, aims to remove the variability of color in faces while keeping the discriminative information.

\section{References}

1. Yip, A., Sinha, P.: Contribution of color to face recognition. Perception 31(5) (2002) 995-1003

2. L. Torres, J.Y.R., Lorente, L.: The importance of the color information in face recognition. In: ICIP. Volume 3. (1999) 627-631

3. III, C.J., Abbott, A.L.: Color face recognition by hypercomplex gabor analysis. In: FG2006, University of Southampton, UK (2006) 126-131

4. Hamilton, W.R.: On a new species of imaginary quantities connected with a theory of quaternions. In: Proc. Royal Irish Academy. Volume 2. (1844) 424-434

5. Zhang, F.: Quaternions and matrices of quaternions. Linear Algebra And Its Applications 251(1-3) (1997) 21-57

6. Pei, S., Cheng, C.: A novel block truncation coding of color images by using quaternion-moment preserving principle. In: ISCAS. Volume 2., Atlanta (USA) (1996) 684-687

7. Sangwine, S., Ell, T.: Hypercomplex fourier transforms of color images. In: ICIP. Volume 1., Thessaloniki (Greece) (2001) 137-140

8. Bihan, N.L., Sangwine, S.J.: Quaternion principal component analysis of color images. In: ICIP. Volume 1., Barcelona (Spain) (2003) 809-812

9. Ja-Han Chang, S.C.P., Ding, J.J.: 2d quaternion fourier spectral analysis and its applications. In: ISCAS. Volume 3., Vancouver (Canada) (2004) 241-244

10. Li, S.Z., Jain, A.K.: 6. In: Handbook of Face Recognition. Springer (2005)

11. Gross, R., Brajovic, V.: An image preprocessing algorithm for illumination invariant face recognition. In: AVBPA, Springer (2003)

12. Lee, K., Ho, J., Kriegman, D.: Nine points of light: Acquiring subspaces for face recognition under variable lighting. In: CVPR. Volume 1. (2001) 519-526

13. Zhang, L., Samaras, D.: Face recognition under variable lighting using harmonic image exemplars. In: CVPR. Volume 1. (2003) 19-25

14. Villegas, M., Paredes, R.: Comparison of illumination normalization methods for face recognition. In: COST 275, University of Hertfordshire, UK (2005) 27-30

15. Turk, M., Pentland, A.: Face recognition using eigenfaces. In: CVPR, Hawaii (1991) 586-591

16. Bihan, N.L., Mars, J.: Subspace method for vector-sensor wave separation based on quaternion algebra. In: EUSIPCO, Toulouse (France) (2002)

17. XM2VTS: http://www.ee.surrey.ac.uk/Reseach/VSSP/xm2vtsdb (CDS00\{1,6\})

18. Luettin, J., Maître, G.: Evaluation protocol for the extended M2VTS database (XM2VTSDB). IDIAP-COM 05, IDIAP (1998) 